\title{
Myeloma prognostic index at diagnosis might be a prognostic marker in patients newly diagnosed with multiple myeloma
}

Dae Sik Kim, Eun Sang Yu, Ka-Won Kang, Se Ryeon Lee, Yong Park, Hwa Jung Sung, Chul Won Choi, and Byung Soo Kim

Division of Oncology and Hematology, Department of Internal Medicine, Korea University School of Medicine, Seoul, Korea

Received: March 1, 2016

Revised : April 13, 2016

Accepted: April 17, 2016

\section{Correspondence to}

Yong Park, M.D.

Division of Oncology and Hematology, Department of Internal Medicine, Korea University School of Medicine, 73 Inchon-ro, Seongbuk-gu, Seoul 02841, Korea Tel: +82-2-920-6847

Fax: +82-2-920-6520

E-mail: paark76@hanmail.net
Background/Aims: The aims of this study were to identify the value of inflammatory markers as pretreatment prognostic factors for patients with multiple myelo$\mathrm{ma}(\mathrm{MM})$ and to estimate the value of a prognostic index including these markers at diagnosis.

Methods: A total of 273 newly diagnosed MM patients undergoing active treatment were analyzed in this study. The prognostic values for survival of the pretreatment inflammatory markers were investigated. A myeloma prognostic index (MPI) was derived using prognostic factors determined to be independently significant on multivariate analysis.

Results: A high pretreatment neutrophil-lymphocyte ratio (NLR), low platelet count, and high C-reactive protein (CRP) level had independently unfavorable significance for overall survival (OS). The MPI was derived based on these factors. Per the MPI, 1 point each was assigned to high NLR, low platelet count, and high CRP. Risk categories were stratified into low- (score o), intermediate- (score 1), and high-risk (score 2 or 3 ) groups. The MPI demonstrated independent statistical significance for OS on multivariate analysis ([intermediate: hazard ratio (HR), 1.91; 95\% confidence interval (CI), 1.12 to 3.24] and [high: HR, 3.37; 95\% CI, 2.00 to 5.69]; $p<0.001)$. Moreover, this significance could be observed regardless of age, renal function, and exposure to novel agents. In addition, the International Staging System risk group could be further significantly stratified using the MPI.

Conclusions: The MPI, consisting of pretreatment inflammatory markers, NLR, platelet count, and CRP, might be effective in predicting the survival of newly diagnosed MM patients undergoing active treatment.

Keywords: Multiple myeloma; Prognosis; Neutrophil-lymphocyte ratio; Platelet count; C-reactive protein

\section{INTRODUCTION}

Multiple myeloma (MM) is a malignant neoplasm of plasma cells derived from a single clonal expansion in the bone marrow (BM) and characterized by bone de- struction, renal failure, anemia, and hypercalcemia [1]. In the United States in 2015, the American Cancer Society estimated that there were 26,850 new cases of MM and 11,240 deaths caused by the disease, and MM accounts for slightly more than $10 \%$ of all hematologic malignan- 
cies [2]. There has been a notable improvement in 5-year survival rates from $25 \%$ in 1977 to $47 \%$ in 2010 since the introduction of novel agents such as bortezomib and lenalidomide into the management of MM [2]. However, patients with MM have highly variable prognoses, and accurate assessment of prognosis is required for optimal treatment. The International Staging System (ISS) is the most widely accepted prognostic scoring system for patients with MM [3].

The relationship between cancer and inflammation has been a subject of interest since Rudolf Virchow noted a connection between inflammation and cancer in 1863 [4]. The systemic inflammation and immune response triggered by cancer are considered critical components of tumor progression, and the tumor microenvironment, largely orchestrated by inflammatory cells, is an indispensable participant in tumor cell proliferation, survival, and migration [5]. Many studies have reported the close relationship between systemic inflammation and cancer $[4,5]$. In this context, several studies have investigated effective markers to measure the systemic inflammatory response in cancer patients, and these markers include C-reactive protein (CRP), albumin, and the neutrophil-lymphocyte ratio (NLR) and platelet-lymphocyte ratio (PLR), among others [6-8].

The NLR is considered worthy of notice. The NLR, simply calculated as neutrophil count $($ cells $/ \mu \mathrm{L})$ divided by lymphocyte count (cells $/ \mu \mathrm{L})$, can be determined from the complete blood count and is known as a marker of systemic inflammation. The NLR was recently identified as a prognostic factor for various types of both solid and hematologic malignancies [9]. In hematologic malignancies, the NLR has been reported to have prognostic value in patients with lymphoma or MM [10-12]. The platelet count, as an acute phase reactant, may reflect the inflammatory response in several types of cancer. Reactive thrombocytosis is associated with poor cancer prognosis, and a high PLR has been identified as a poor prognostic factor in various solid tumors [6]. CRP is also widely known as a major inflammatory marker, and the association between CRP and cancer prognosis has been demonstrated in previous studies [13]. The prognostic role of CRP has also been revealed in hematologic malignancies such as MM [14].

The aims of the current study were to identify the value of inflammatory markers as pretreatment prognostic factors for patients with MM and to estimate the value of a prognostic index including these markers at diagnosis.

\section{METHODS}

Patients who were newly diagnosed with MM between September 2001 and December 2013 at three tertiary hospitals (Korea University Anam Hospital, Korea University Guro Hospital, and Korea University Ansan Hospital) of Korea University Medical Center were analyzed. The diagnosis of MM was based on International Myeloma Working Group (IMWG) criteria 2014. Patients who were diagnosed with monoclonal gammopathy of undetermined significance, smoldering myeloma, solitary plasmacytoma, Waldenstrom's macroglobulinemia, or primary plasma cell leukemia were excluded. All patients were actively treated with chemotherapy including novel agents such as thalidomide, lenalidomide, and bortezomib. Transplant-eligible patients were treated with an autologous stem cell transplantation after induction chemotherapy.

Before treatment, all patients were screened by age, sex, and Eastern Cooperative Oncology Group performance status (PS). Clinical features at the time of diagnosis including levels of serum and urine M-protein, percentage of BM plasma cells, the presence of osteolytic bone lesions, and hemodialysis were analyzed. Baseline laboratory evaluations including hemoglobin level, total white blood cell count and differential count, platelet count, serum albumin, serum $\beta 2$-microglobulin $(\beta 2-\mathrm{mg})$, serum calcium, serum creatinine, CRP, and serum lactate dehydrogenase were performed to evaluate the pre-chemotherapy status and risk. Cytogenetic analysis of BM specimens was performed using conventional cytogenetic protocols and interphase fluorescence in situ hybridization (FISH). For conventional cytogenetics, at least 20 metaphases were analyzed in each case. In our institute, the FISH panel for MM including IGH (14932) break apart, translocation of chromosomes 4 and $14(\mathrm{t}(4 ; 14))$, translocation of chromosomes 14 and 16 $(t(14 ; 16))$, deletion of $13 q 14$ and deletion of $17 p 13$ had been used since 2008. According to IMWG consensus criteria 2014, we considered cytogenetically detected 17p deletion, and chromosome 14 translocation to indicate poor risk. In FISH analysis, t(4;14), t(14;16), and del(17p13) were 
also considered poor-risk cytogenetics [15,16]. Prognostic factors for overall survival (OS) were determined via univariate and multivariate analysis. A prognostic index was designed using the variables that were the most significant prognostic factors on multivariate analysis. Subgroups divided by age, renal function, and use of novel agents were analyzed. This study was approved by the Korea University Hospital Institutional Review Board.

\section{Statistical analysis}

Data analysis was performed using the IBM SPSS version 20.0 (IBM Co., Armonk, NY, USA). Differences among treatment groups were compared using the Student $t$ test and the chi-square test. OS was defined as the time interval from the date of diagnosis to the date of death. OS was calculated using the Kaplan-Meier method. Univariate and multivariate analysis for prognostic factors for OS were performed using the log-rank test and Cox proportional hazards method with the following variables: age, PS, M-protein, BM plasma cells, cytogenetic risk, bone lesions, NLR, platelet count, CRP level, $\beta 2-\mathrm{mg}$, serum lactate dehydrogenase, serum calcium, serum creatinine, and use of novel agents. $p$ values $<0.05$ were considered significant.

\section{RESULTS}

\section{Patient selection and baseline characteristics}

A total of 273 patients newly diagnosed with MM were enrolled in this study. Among them, 87 were treated with only conventional chemotherapy without novel agents. This treatment included high-dose dexamethasone, melphalan plus prednisone or vincristine, adriamycin, and dexamethasone. A total of 186 patients received more than one novel agent during the course of treatment (141 patients received bortezomib, 117 patients received thalidomide, and 10 patients received lenalidomide). The median age of all patients at the time of diagnosis was 64 years (range, 30 to 83). The immunoglobulin (Ig) subtypes were IgG (70.1\%), IgA (21\%), and others $(2.6 \%)$, and 17 patients $(6.3 \%)$ secreted only free light chain. At diagnosis, 59.0\% of patients had anemia (hemoglobin < $10.0 \mathrm{~g} / \mathrm{dL}$ ), 16.3\% had hypercalcemia (serum calcium $>11.0 \mathrm{mg} / \mathrm{dL}$ ), $23.3 \%$ had renal dysfunction (serum creatinine $>2.0 \mathrm{mg} / \mathrm{dL}$ ), and $81.3 \%$ had bone le- sions. Table 1 shows the patients' baseline characteristics. There were no significant differences between the groups that received conventional chemotherapy and those that received novel agents. However, slightly more patients in the novel agent group than in the conventional chemotherapy group received stem cell transplantation (22.2\% vs. $6.9 \%, p=0.002)$.

Cytogenetic abnormalities were investigated in 252 patients using a conventional chromosome study and in 116 patients using FISH. Chromosomal abnormalities were found in $33.7 \%(85 / 252)$ of the patients. The incidence of the complex karyotype, which has more than two abnormalities, was $27.8 \%$ (70/252). Hyperdiploidy was detected in $11.5 \%$ (29/252) of the patients and hypodiploidy was detected in 9.1\%. A 14932 rearrangement was detected using FISH in 45 patients (38.8\%), which most commonly included IGH break apart (21.6\%) followed by $t(4 ; 14)$ and $t(14 ; 16)(11.2 \%$, and $6.0 \%$, respectively). Deletion of p53 (17p13) and deletion of Rb1 (13q14) were found in $22.4 \%$ and $19.0 \%$ of patients, respectively.

Rates of complete or near complete response with initial treatment were higher in the group receiving bortezomib containing chemotherapy than thalidomide based group or conventional chemotherapy group $(17.9 \%, 14.6 \%, 7.8 \%, p=0.046)$. In addition, the rates of very good partial response or better in the bortezomib group were significantly higher than other groups ( $42.9 \%$ vs. $35.4 \%$ vs. $22.8 \%, p=0.009$ ).

\section{Prognostic factors of survival}

First, we performed univariate analysis to identify the prognostic factors for OS. On univariate analysis for OS, age $\geq 65(p=0.001), \mathrm{PS} \geq 2(p<0.001)$, high serum Mprotein $(p=0.024)$, high BM plasma cells $(p<0.001)$, cytogenetic high risk $(p<0.001)$, high NLR $(p<0.001)$, low platelet count $(p<0.001)$, elevated CRP $(p<0.001)$, low albumin $(p=0.009), \beta 2-\mathrm{mg}(p<0.001)$, use of novel agents $(p=0.004)$, and stem cell transplantation $(p=0.001)$ were statistically significant prognostic factors (Table 2). Multivariate analysis for OS showed that $\mathrm{PS} \geq 2(p=0.009)$, high BM plasma cells $(p<0.001)$, cytogenetic high risk $(p=0.007)$, high NLR $(p=0.019)$, low platelet count $(p=$ 0.047), elevated CRP ( $p=0.002)$, use of novel agents $(p<$ $0.001)$, and stem cell transplantation $(p=0.05)$ were independently significant prognostic factors (Table 2). 
Table 1. Patients' baseline characteristics and treatment response

\begin{tabular}{|c|c|c|c|c|}
\hline Characteristic & Total $(\mathrm{n}=273)$ & $\begin{array}{l}\text { Conventional chemotherapy } \\
\qquad(\mathrm{n}=87)\end{array}$ & $\begin{array}{l}\text { Novel agents } \\
\quad(\mathrm{n}=186)\end{array}$ & $p$ value \\
\hline Age, yr & $64(30-83)$ & $65(34-79)$ & $62(30-83)$ & $0.705^{\mathrm{a}}$ \\
\hline Sex, male/female & $160 / 113$ & $49 / 38$ & $111 / 75$ & $0.600^{b}$ \\
\hline ECOG PS $\geq 2$ & $57(21.2)$ & $21(24 \cdot 4)$ & $36(19 \cdot 7)$ & $0.374^{b}$ \\
\hline Monoclonal protein & & & & $0.177^{\mathrm{b}}$ \\
\hline $\operatorname{IgG}$ & $190(70.1)$ & $63(72.4)$ & $127(69)$ & \\
\hline $\operatorname{IgA}$ & $57(21.0)$ & $21(24.1)$ & $36(19.6)$ & \\
\hline $\operatorname{IgD}$ or IgE & $7(2.6)$ & $1(1.1)$ & $6(3 \cdot 3)$ & \\
\hline Light chain disease & $17(6.3)$ & $2(2.3)$ & $15(8.2)$ & \\
\hline International Staging System & & & & $0.955^{\mathrm{b}}$ \\
\hline 1 & $56(20.5)$ & $18(20.7)$ & $38(20.4)$ & \\
\hline 2 & $110(40.3)$ & $36(41.4)$ & $74(39.8)$ & \\
\hline 3 & $107(39.2)$ & $33(37 \cdot 9)$ & $74(39.8)$ & \\
\hline Serum M-protein $\geq 3.0 \mathrm{~g} / \mathrm{dL}$ & $122(47.1)$ & $44(51.8)$ & $78(44.8)$ & $0.294^{b}$ \\
\hline BM plasma cells $\geq 40 \%$ & $117(45 \cdot 7)$ & $37(45 \cdot 1)$ & $80(46.0)$ & $0.778^{b}$ \\
\hline Hemoglobin < $10.0 \mathrm{~g} / \mathrm{dL}$ & $157(59.0)$ & $56(64 \cdot 4)$ & $101(56.4)$ & $0.217^{\mathrm{b}}$ \\
\hline Serum calcium > $11.0 \mathrm{mg} / \mathrm{dL}$ & $31(11.8)$ & $8(9.4)$ & $23(12.9)$ & $0.409^{b}$ \\
\hline Serum creatinine $>2.0 \mathrm{mg} / \mathrm{dL}$ & $62(23 \cdot 3)$ & $22(25 \cdot 3)$ & $40(22.3)$ & $0.595^{\mathrm{b}}$ \\
\hline Bone lesion & $208(81.3)$ & $63(75 \cdot 9)$ & $145(83.8)$ & $0.129^{\mathrm{b}}$ \\
\hline Neutrophil count, $\times 10^{9} / \mathrm{L}$ & $3.5(0.4-27.7)$ & $4.0(0.5-27.7)$ & $3.2(0.4-12.1)$ & $0.016^{\mathrm{a}}$ \\
\hline Lymphocyte count, $\times 10^{9} / \mathrm{L}$ & $1.8(0.3-8.70)$ & $1.7(0.3-5.5)$ & $1.7(0.4-8.7)$ & $0.947^{\mathrm{a}}$ \\
\hline Platelet count, $\times 10^{9} / \mathrm{L}$ & $186(15-744)$ & $173(28-496)$ & $186(15-744)$ & $0.308^{\mathrm{a}}$ \\
\hline Serum LDH > upper limit of normal & $66(25 \cdot 9)$ & $25(29.8)$ & $41(24.0)$ & $0.322^{b}$ \\
\hline Cytogenetics risk & & & & $0.163^{b}$ \\
\hline Standard & $180(71.4)$ & $59(79.7)$ & $121(68.0)$ & \\
\hline High & $72(28.6)$ & $15(20.3)$ & $57(32.0)$ & \\
\hline Stem cell transplant & $47(17 \cdot 3)$ & $6(6.9)$ & $41(22.2)$ & $0.002^{b}$ \\
\hline
\end{tabular}

Values are presented as median (range) or number (\%).

ECOG PS, Eastern Cooperative Oncology Group performance status; Ig, immunoglobulin; BM, bone marrow; LDH, lactate dehydrogenase.

${ }^{\mathrm{a}}$ Student $t$ test. ${ }^{\mathrm{b}}$ Chi-square test.

\section{Derivation of myeloma prognostic index}

The myeloma prognostic index (MPI) was designed using the variables that are associated with inflammation among those found to be significant prognostic factors for OS on multivariate analysis. Pretreatment high NLR ( $\geq 2.25$ ), low platelet count $(<150,000$ cells $/ \mu \mathrm{L})$, and high CRP ( $\geq 5 \mathrm{mg} / \mathrm{L}$ ) had unfavorable significance for OS independent of other prognostic factors. We used these variables to construct a MPI for prognosis. Our prognostic index assigned 1 point each to high NLR, low platelet count, and high CRP. After the final score was obtained (maximum score of 3), risk categories were stratified into low (score o), intermediate (score 1), and high (score 2 or 3 ) risk groups, respectively. This MPI showed statistical significance for OS on univariate and multivariate analysis $(p<0.001$ ) (Table 3 ). The median OS was significantly different among risk groups by MPI as well as ISS (Fig. 1A, MPI: low risk, 6.21 years; intermediate risk, 2.43 years; high risk, 1.01 years; $p<0.001$ ) (Fig. 1B, ISS: low risk, 4.91 years; intermediate risk, 2.7 years; high 
Kim DS, et al. Myeloma prognostic index

Table 2. Prognostic factors for overall survival

\begin{tabular}{|c|c|c|c|c|c|}
\hline \multirow{2}{*}{ Factor } & \multicolumn{2}{|c|}{ Univariate analysis } & \multicolumn{3}{|c|}{ Multivariate analysis } \\
\hline & OS, mon & $p$ value $^{\mathrm{a}}$ & HR & $95 \% \mathrm{CI}$ & $p$ value $^{\mathrm{b}}$ \\
\hline Age, yr & & 0.001 & & & \\
\hline$<65$ & 45.2 & & & & \\
\hline$\geq 65$ & 22.2 & & & & \\
\hline Performance status & & $<0.001$ & & & 0.009 \\
\hline $0-1$ & 42.0 & & 1 & & \\
\hline$\geq 2$ & 13.0 & & 1.73 & $1.15-2.60$ & \\
\hline Serum M-protein, g/dL & & 0.024 & & & \\
\hline$<3.0$ & $39 \cdot 7$ & & & & \\
\hline$\geq 3.0$ & 22.2 & & & & \\
\hline BM plasma cell, \% & & $<0.001$ & & & $<0.001$ \\
\hline$<40$ & 38.0 & & 1 & & \\
\hline$\geq 40$ & 20.2 & & 1.89 & $1.32-2.69$ & \\
\hline Cytogenetic risk & & $<0.001$ & & & 0.007 \\
\hline Standard & 45.2 & & 1 & & \\
\hline High & $15 \cdot 3$ & & 1.71 & $1.16-2.53$ & \\
\hline NLR & & $<0.001$ & & & 0.019 \\
\hline$<2.25$ & 44.2 & & 1 & & \\
\hline$\geq 2.25$ & 16.0 & & 1.56 & $1.08-2.27$ & \\
\hline Platelets, cells $/ \mu \mathrm{L}$ & & $<0.001$ & & & 0.047 \\
\hline$\geq 150,000$ & 45.2 & & 1 & & \\
\hline$<150,000$ & 18.5 & & 1.48 & $1.01-2.18$ & \\
\hline CRP, mg/L & & $<0.001$ & & & 0.002 \\
\hline$<5.0$ & $47 \cdot 7$ & & 1 & & \\
\hline$\geq 5.0$ & $15 \cdot 3$ & & 1.82 & $1.25-2.64$ & \\
\hline Albumin, g/dL & & 0.009 & & & \\
\hline$\geq 3.5$ & 39.7 & & & & \\
\hline$<3.5$ & $23 \cdot 3$ & & & & \\
\hline Serum creatinine, $\mathrm{mg} / \mathrm{dL}$ & & 0.066 & & & \\
\hline$\leq 2.0$ & 32.9 & & & & \\
\hline$>2.0$ & 16.8 & & & & \\
\hline$\beta 2-\mathrm{mg}, \mathrm{mg} / \mathrm{L}$ & & $<0.001$ & & & \\
\hline$<3.5$ & 47.1 & & & & \\
\hline$\geq 3.5$ and $<5.5$ & 32.9 & & & & \\
\hline$\geq 5 \cdot 5$ & 18.1 & & & & \\
\hline Use of novel agent & & 0.004 & & & $<0.001$ \\
\hline No & 14.9 & & 2.07 & $1.41-3.04$ & \\
\hline Yes & 38.0 & & 1 & & \\
\hline Stem cell transplant & & 0.001 & & & 0.050 \\
\hline No & 26.3 & & 1.82 & $1.00-3.32$ & \\
\hline Yes & 69.4 & & 1 & & \\
\hline
\end{tabular}

OS, overall survival; HR, hazard ratio; CI, confidence interval; BM, bone marrow; NLR, neutrophil-leukocyte ratio; CRP, Creactive protein.

${ }^{\mathrm{a}}$ Log-rank test. ${ }^{\mathrm{b}}$ Cox proportional hazards model. 
Table 3. Multivariate analysis including myeloma prognostic index for overall survival

\begin{tabular}{|c|c|c|c|}
\hline \multirow{2}{*}{ Factor } & \multicolumn{3}{|c|}{ Multivariate analysis } \\
\hline & HR & $95 \% \mathrm{CI}$ & $p$ value \\
\hline Performance status & & & 0.003 \\
\hline $0-1$ & 1 & & \\
\hline$\geq 2$ & 1.82 & $1.22-2.73$ & \\
\hline BM plasma cell, $\%$ & & & 0.001 \\
\hline$<40$ & 1 & & \\
\hline$\geq 40$ & 1.82 & $1.29-2.58$ & \\
\hline Cytogenetic risk & & & 0.002 \\
\hline Standard & 1 & & \\
\hline High & 1.82 & $1.25-2.65$ & \\
\hline Myeloma prognostic index & & & $<0.001$ \\
\hline Low & 1 & & \\
\hline Intermediate & 1.91 & $1.12-3.24$ & \\
\hline High & $3 \cdot 37$ & $2.00-5.69$ & \\
\hline Use of novel agents & & & $<0.001$ \\
\hline No & 2.15 & $1.46-3.17$ & \\
\hline Yes & 1 & & \\
\hline Stem cell transplant & & & 0.040 \\
\hline No & 1.87 & $1.03-3.38$ & \\
\hline Yes & 1 & & \\
\hline
\end{tabular}

The multivariate analysis results including myeloma prognostic index (MPI) with prior significant prognostic factors. The MPI achieved statistical significance in multivariate analysis.

$\mathrm{HR}$, hazard ratio; CI, confidence interval; BM, bone marrow.
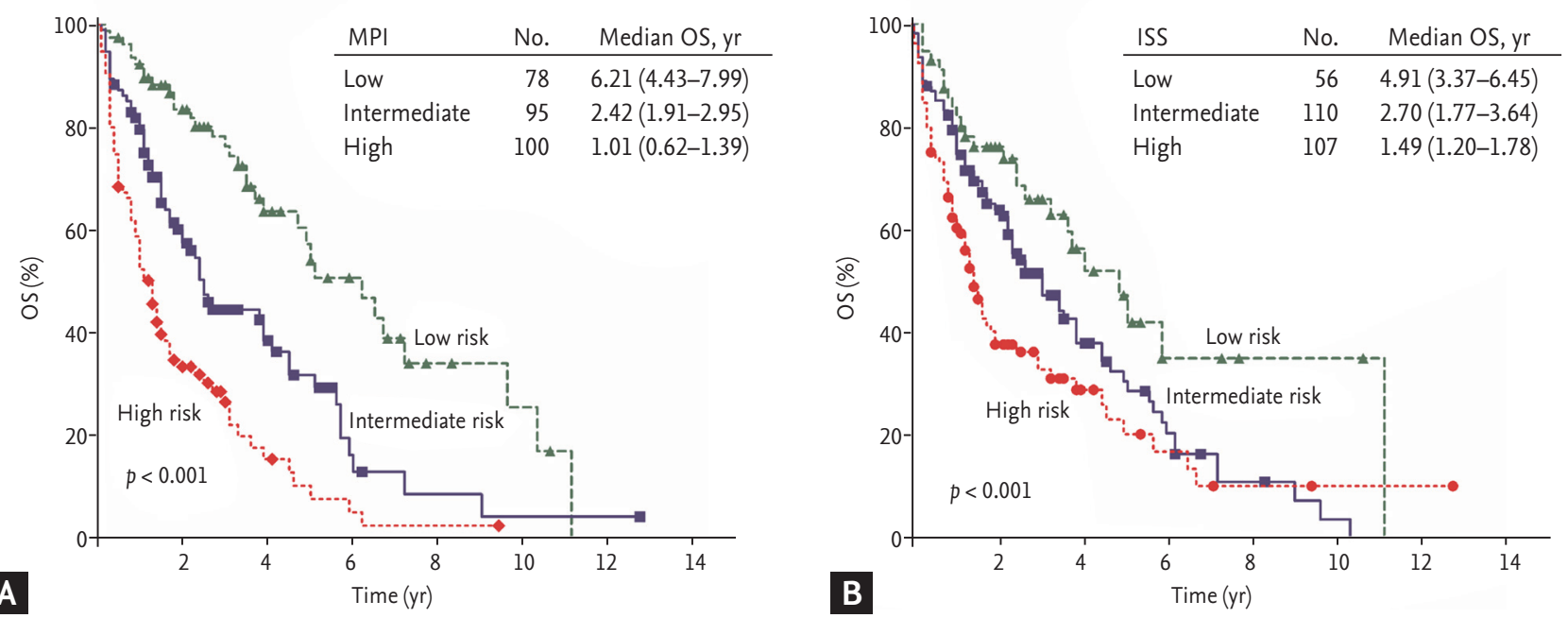

Figure 1. Survival curve according to scoring system in all patients. Both scoring systems showed statistical significance. (A) Myeloma prognostic index (MPI). (B) International Staging System (ISS). OS, overall survival. 

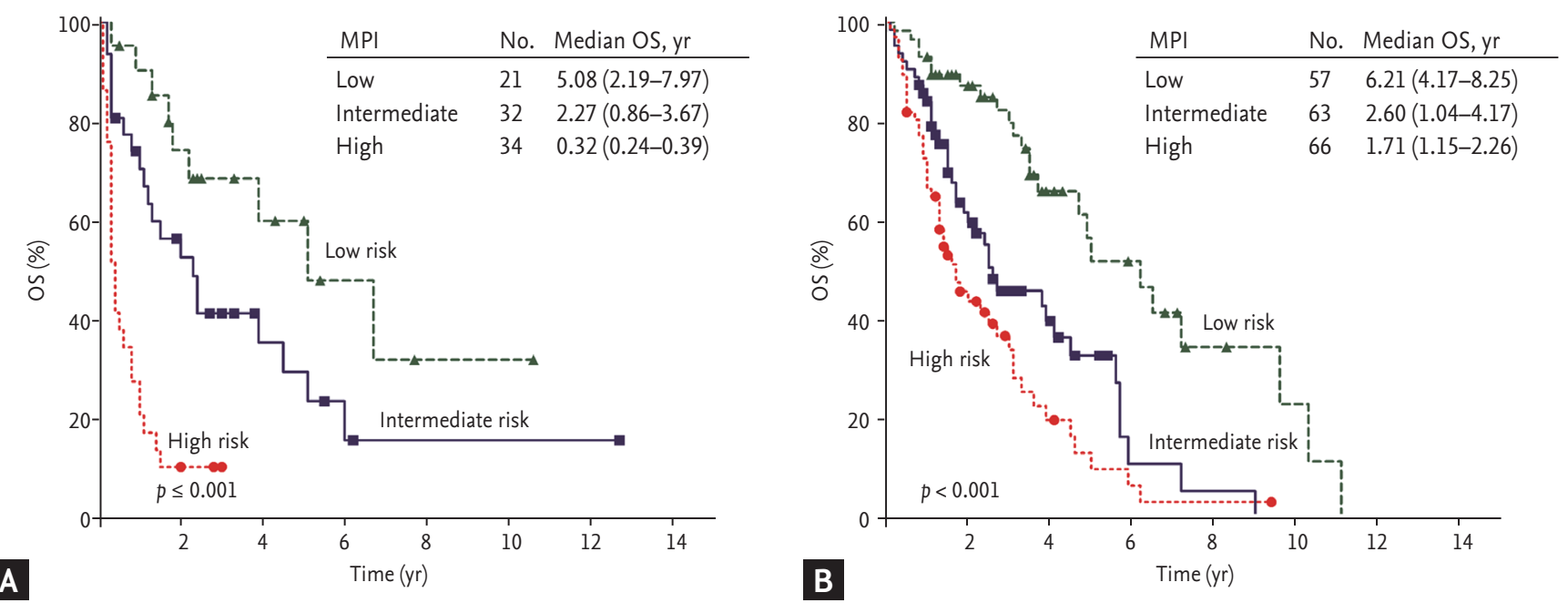

Figure 2. Survival curve by subgroups divided by treatment strategies. (A) Conventional chemotherapy. (B) Novel agents. MPI, myeloma prognostic index; OS, overall survival.
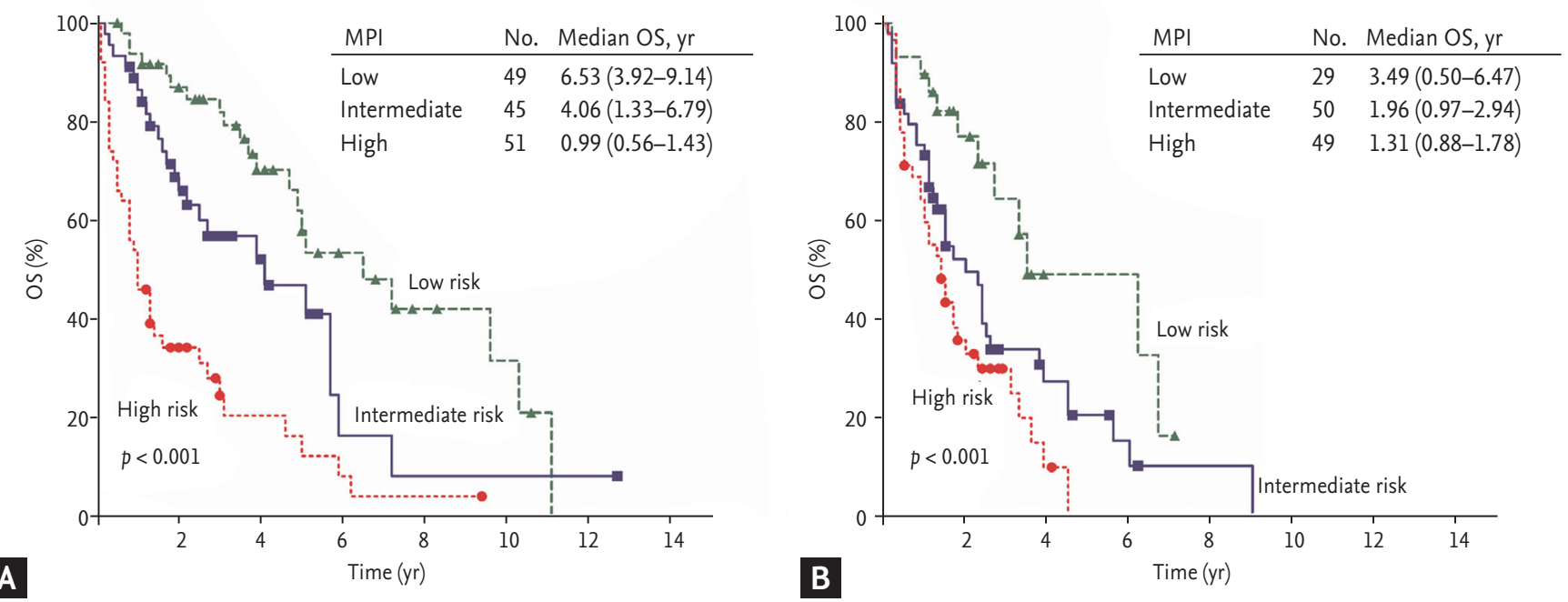

Figure 3. Survival curve by subgroups divided by age. (A) Young age group (age $<65$ years, transplant candidates). (B) Older age group (age $\geq 65$ years, non-transplant candidates). MPI, myeloma prognostic index; OS, overall survival.

risk, 1.49 years; $p<0.001)$.

In the subgroup analysis, the MPI was suitable for risk stratification in patients treated with novel agents as well as in patients treated with conventional chemotherapy (Fig. 2). In the subgroup analysis according to age, both the young age group (age $<65$ years, transplant candidates) and the older age group (age $\geq 65$ years, non-transplant candidates) could be clearly stratified by the MPI (Fig. 3). Our index also demonstrated effective risk stratification in patients with abnormal serum cre- atinine levels (serum creatinine level > 2 mg/dL) (Fig. 4).

Moreover, we were able to further stratify the ISS risk categories using this inflammation index. When the MPI was applied to each ISS risk category group, the patients in each ISS risk group were subdivided into three risk groups. The patients were adequately distributed among the subgroups, and the risk stratification in all three ISS subgroup showed statistical significance (ISS I, $p<0.001$; ISS II, $p=0.013$; and ISS III, $p<0.001$ ) (Fig. 5). 

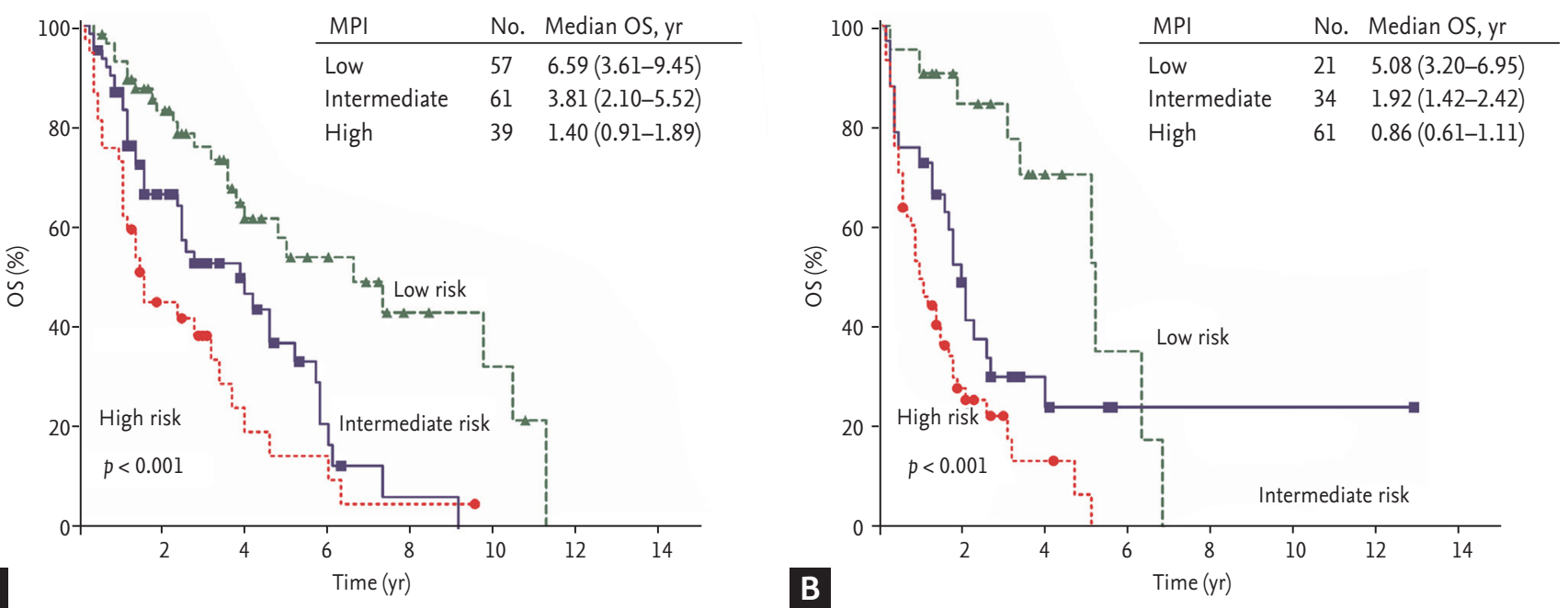

Figure 4. Survival curve by subgroup divided by renal function. (A) Normal renal function group (creatinine $\leq 2.0)$. (B) Abnormal renal function group (creatinine > 2.0). MPI, myeloma prognostic index; OS, overall survival.

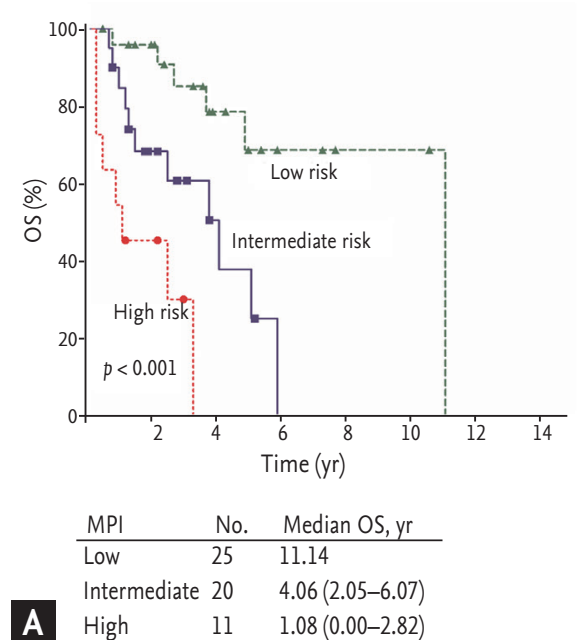

Figure 5. Survival curve of myeloma prognostic index in patients with International Staging System (ISS) subcategories. (A) ISS category I, (B) ISS category II, and (C) ISS category III. MPI, myeloma prognostic index; OS, overall survival.

\section{DISCUSSION}

This study was designed to identify the clinical significance of factors reflecting inflammation and a prognostic index composed of these factors for patients with newly diagnosed MM. The MPI, which consists of pretreatment inflammatory markers including NLR, platelet count, and CRP, might be effective to stratify risk groups and predict survival. In fact, it showed prognostic significance regardless of age, renal function, and exposure to novel agents. Moreover, considering that the prognosis of patients could be stratified by the MPI within each subgroup according to the ISS, the MPI could be considered to complement the ISS.

The relationship between inflammation and cancer has been widely investigated in many studies $[5,17]$. In summary, there are some kinds of inflammation that provide the tumor with an environment favorable for survival. Inflammatory cells in the tumor microenvironment such as macrophages, dendritic cells, and lymphocytes are known to be involved in this process by stimulating tumor cell growth, progression, angiogenesis, and 
metastasis. In addition, proinflammatory cytokines and chemokines found in cancer patients also contribute directly to malignant progression and promote resistance to anti-cancer therapy [4]. That is, the balances between proinflammatory cytokines and anti-inflammatory cytokines in cancer patients could have an effect on the level of protumorous inflammation, contributing to the neoplastic outcome [5]. In these contexts, there have been efforts to investigate effective markers to reflect the protumorous inflammatory status in cancer patients. The NLR and CRP are representative examples.

The physiological response of the innate immune system to systemic inflammation is clinically presented as neutrophilia and relative lymphopenia [7]. Neutrophilia in systemic inflammation is induced by demargination of neutrophils, stimulation of progenitor cells by growth factors and delayed apoptosis of neutrophils. The mechanisms responsible for lymphopenia include redistribution of lymphocytes within the lymphatic system and prominently accelerated apoptosis [7]. The NLR directly reflects the balance between neutrophilia and lymphopenia. As an elevated NLR has been recognized as a poor prognostic factor in various solid cancers and hematologic malignancies [9-11], the NLR is considered to be a marker reflecting the degree of protumorous inflammatory status.

CRP is known as an acute phase reactant, which reflects tissue injury and systemic inflammation. Currently, an elevated CRP level is known to be an adverse prognostic factor in various cancers including MM $[14,18]$. The association between CRP and cancer prognosis could be explained in various ways. First, tumor growth can directly cause inflammation of surrounding tissue, and this leads to increases in acute phase proteins including CRP [4]. Second, proinflammatory cytokines such as interleukin 1 , interleukin 6, and tumor necrosis factor are known to stimulate the synthesis of CRP in cancer patients [19]. That is, elevated CRP levels reflect increased concentrations of proinflammatory cytokines. Moreover, circulating CRP acts directly on tumor cells and contributes to tumor progression [20], and therefore, elevated levels of CRP in cancer patients might reflect the aggressiveness of the tumor itself.

Generally, thrombocytosis, a marker of systemic inflammation, is associated with poor outcomes in various solid cancers. Thus, a high PLR has been reported as a poor prognostic marker for OS in several malignancies [6]. However, contrary to the findings in solid cancers, a low platelet count or inverse PLR have been demonstrated to be a poor prognostic factor in patients with MM [18,21,22]. This difference between MM and other solid cancers may be due to the anatomical features of MM, which develops from the BM. That is, thrombocytopenia is considered to directly reflect the tumor burden because normal thrombopoiesis is gradually inhibited as malignant plasma cells accumulate in the $\mathrm{BM}$. In addition to the anatomical features of MM, inflammatory cytokines are considered to be involved in decreased thrombopoiesis in MM. For example, transforming growth factor- $\beta 1$, which is known to be secreted by malignant plasma cells and to mediate plasma cell growth and survival [23], has been shown to arrest the maturation of megakaryocyte colony-forming units [24]. Moreover, the half-life of platelets has been reported to be significantly reduced in patients with MM [25]. Therefore, thrombocytopenia might reflect the adverse prognosis of MM by the combined effect of anatomical location, inhibitory cytokines, and increased turnover.

Recently, a consensus has arisen that cytogenetic abnormalities play an important and independent role in risk stratification in MM patients. As a result, the revised ISS, which incorporates the cytogenetics into the ISS, improved the prognostic significance of the ISS $[15,16]$. In this context, the results of the current study suggest the possibility that the MPI can show a synergistic effect with the ISS in predicting the prognosis of patients with MM. First, the MPI is based on biologic features focusing on the host immune response (NLR and CRP), the tumor burden, and the interaction between the tumor and the microenvironment (platelet count). These features are considered to be not fully reflected in the ISS. In fact, the present study revealed that the survival for each ISS group could be further stratified by each MPI group (Fig. 5). Another potential benefit of the MPI is that it showed prognostic significance for all subgroups of patients regardless of age, renal function, and exposure to novel agents. However, a firm conclusion regarding the MPI cannot be drawn owing to the limitations of this study. The MPI was based on retrospective analysis and was not validated in an independent cohort. In addition, the effects with regard to progression-free survival were not analyzed because of heterogeneous induction 
treatments. In conclusion, the MPI using the NLR, CRP, and platelet count might have prognostic significance for patients with newly diagnosed MM regardless of age, renal function, and exposure to novel agents, and might function synergistically with the ISS or revised ISS. For confirmatory results, validation in an independent cohort will be required.

\section{KEY MESSAGE}

1. Myeloma prognostic index (MPI) composed of the neutrophil-lymphocyte ratio, C-reactive protein, and the platelet count could predict the survival of newly diagnosed myeloma patients.

2. MPI could be effective in predicting patients' survival regardless of age, renal function, and exposure to novel agents.

3. We suggest the possibility that this model could function synergistically with the International Staging System.

\section{Conflict of interest}

No potential conflict of interest relevant to this article was reported.

\section{Acknowledgments}

This research was supported by a grant from the Korea Health Technology R\&D Project through the Korea Health Industry Development Institute (KHIDI), funded by the Ministry of Health and Welfare, Republic of Korea (grant number: HI14C2750).

\section{REFERENCES}

1. Kyle RA, Rajkumar SV. Multiple myeloma. Blood 2008; 111:2962-2972.

2. Siegel RL, Miller KD, Jemal A. Cancer statistics, 2015. CA Cancer J Clin 2015;65:5-29.

3. Greipp PR, San Miguel J, Durie BG, et al. International Staging System for multiple myeloma. J Clin Oncol 2005;23:3412-3420.

4. Balkwill F, Mantovani A. Inflammation and cancer: back to Virchow? Lancet 2001;357:539-545.

5. Coussens LM, Werb Z. Inflammation and cancer. Nature
2002;420:860-867.

6. Templeton AJ, Ace O, McNamara MG, et al. Prognostic role of platelet to lymphocyte ratio in solid tumors: a systematic review and meta-analysis. Cancer Epidemiol Biomarkers Prev 2014;23:1204-1212.

7. Zahorec R. Ratio of neutrophil to lymphocyte counts: rapid and simple parameter of systemic inflammation and stress in critically ill. Bratisl Lek Listy 2001;102:5-14.

8. McMillan DC. The systemic inflammation-based Glasgow Prognostic Score: a decade of experience in patients with cancer. Cancer Treat Rev 2013;39:534-540.

9. Templeton AJ, McNamara MG, Seruga B, et al. Prognostic role of neutrophil-to-lymphocyte ratio in solid tumors: a systematic review and meta-analysis. J Natl Cancer Inst 2014;106:dju124.

10. Keam B, Ha H, Kim TM, et al. Neutrophil to lymphocyte ratio improves prognostic prediction of International Prognostic Index for patients with diffuse large B-cell lymphoma treated with rituximab, cyclophosphamide, doxorubicin, vincristine and prednisone. Leuk Lymphoma 2015;56:2032-2038.

11. Kelkitli E, Atay H, Cilingir F, et al. Predicting survival for multiple myeloma patients using baseline neutrophil/ lymphocyte ratio. Ann Hematol 2014;93:841-846.

12. Koh YW, Kang HJ, Park C, et al. Prognostic significance of the ratio of absolute neutrophil count to absolute lymphocyte count in classic Hodgkin lymphoma. Am J Clin Pathol 2012;138:846-854.

13. Shrotriya S, Walsh D, Bennani-Baiti N, Thomas S, Lorton C. C-reactive protein is an important biomarker for prognosis tumor recurrence and treatment response in adult solid tumors: a systematic review. PLoS One 2015;10:e0143080.

14. Bataille R, Boccadoro M, Klein B, Durie B, Pileri A. C-reactive protein and beta-2 microglobulin produce a simple and powerful myeloma staging system. Blood 1992;80:733737.

15. Palumbo A, Avet-Loiseau H, Oliva S, et al. Revised International Staging System for multiple myeloma: a report from International Myeloma Working Group. J Clin Oncol 2015;33:2863-2869.

16. Chng WJ, Dispenzieri A, Chim CS, et al. IMWG consensus on risk stratification in multiple myeloma. Leukemia 2014;28:269-277.

17. Hanahan D, Weinberg RA. Hallmarks of cancer: the next generation. Cell 2011;144:646-674. 
18. Barlogie B, Bolejack V, Schell M, Crowley J. Prognostic factor analyses of myeloma survival with intergroup trial S9321 (INT 0141): examining whether different variables govern different time segments of survival. Ann Hematol 2011;90:423-428.

19. Black S, Kushner I, Samols D. C-reactive protein. J Biol Chem 2004;279:48487-48490.

20. Wang CS, Sun CF. C-reactive protein and malignancy: clinico-pathological association and therapeutic implication. Chang Gung Med J 2009;32:471-482.

21. Kim SJ, Kim K, Kim BS, et al. Clinical features and survival outcomes in patients with multiple myeloma: analysis of web-based data from the Korean Myeloma Registry. Acta Haematol 2009;122:200-210.

22. Jung SH, Kim JS, Lee WS, et al. Prognostic value of the inverse platelet to lymphocyte ratio (iPLR) in patients with multiple myeloma who were treated up front with a novel agent-containing regimen. Ann Hematol 2016;95:55-61.

23. Cook G, Campbell JD, Carr CE, Boyd KS, Franklin IM. Transforming growth factor beta from multiple myeloma cells inhibits proliferation and IL-2 responsiveness in T lymphocytes. J Leukoc Biol 1999;66:981-988.

24. Sakamaki S, Hirayama Y, Matsunaga T, et al. Transforming growth factor-betar (TGF-betal) induces thrombopoietin from bone marrow stromal cells, which stimulates the expression of TGF-beta receptor on megakaryocytes and, in turn, renders them susceptible to suppression by TGF-beta itself with high specificity. Blood 1999;94:19611970.

25. Fritz E, Ludwig H, Scheithauer W, Sinzinger H. Shortened platelet half-life in multiple myeloma. Blood 1986;68:514520. 